\title{
A method for the purification of bacterial flagellin that allows simple upscaling
}

\author{
Yanina Hiriart • Agustina Errea • \\ Dolores González Maciel · Juan Carlos Lopez • \\ Martin Rumbo
}

Received: 8 July 2010/ Accepted: 9 May 2011/Published online: 22 May 2011

(C) Springer Science+Business Media B.V. 2011

\begin{abstract}
There is a growing interest in enterobacterial flagellins that may result in a demand to produce flagellin on an industrial scale for possible applications as an adjuvant, immunomodulatory agent or vaccine antigen. Traditionally, small-scale production of flagellin has occurred in the laboratory by flagellar shearing of bacterial surfaces and subsequent ultracentrifugation. The main drawback of this method is the need to use low-agitation cultures to avoid the loss of flagella due to shearing during culture. In the present work, we describe a scalable protocol for the production of flagellin with higher yields than traditional laboratory-scale protocols. The use of crossflow filtration to concentrate bacterial cultures combines extensive shearing of flagella with a reduction in volume, greatly simplifying downstream processing. This technique also allows the use of highly-agitated culture conditions because any sheared flagella are retained in the bacterial concentrate. Flagella obtained with this procedure showed in vivo and in vitro innate activating capacities similar to those of flagella produced at laboratory scale. This procedure is flexible, allowing an increase in production scale, an enhancement of flagellin yield and no requirement for expensive equipment.
\end{abstract}

Keywords Flagellin · Scaling up · Cross-flow filtration · Innate response

Y. Hiriart · A. Errea · D. González Maciel · M. Rumbo ( $₫)$ Laboratorio de Investigaciones del Sistema Inmune (LISIN). Facultad de Ciencias Exactas, Universidad Nacional de La Plata, 47 y 115 (1900), La Plata, Argentina

e-mail: martinr@biol.unlp.edu.ar

Y. Hiriart · J. C. Lopez

Instituto Biológico Argentino (BIOL) S.A.I.C, Buenos Aires, Argentina

\section{Introduction}

Flagellin is the main structural component of bacterial flagella and is a potent agonist of innate immunity through interactions with the membrane-bound receptor TLR5 and the cytosolic receptors IPAF and Naip5 (Miao et al. 2007; Lightfield et al. 2008). Due to its multiple roles in enhancing innate responses, flagellin has been proposed as a mucosal or systemic adjuvant to enhance the efficacy of immunization strategies in different experimental settings, including enhancing the efficacy of an anti-influenza mucosal vaccination (Lee et al. 2006; Strindelius et al. 2004; Honko et al. 2006; Skountzou et al. 2010). Furthermore, because flagellin induces strong granulocyte-monocyte colony-stimulating factor (GM-CSF) expression when delivered by the systemic route, it has been proposed as a booster for the recovery from neutropaenia induced by anti-neoplasic chemotherapy or radiotherapy (Burdelya et al. 2008). Flagellum-dependent motility is an important virulence factor of several pathogens (Lane et al. 2007; Rumbo et al. 2006). Flagellin has also been demonstrated to be critical for bovine intestinal colonization and for adhesion to epithelial cells by enterohaemorrhagic Escherichia. coli (Mahajan et al. 2009) and has been proposed as a vaccine antigen to prevent colonization of cattle by flagella-expressing EHEC (McNeilly et al. 2008).

Due to increasing applications in biotechnology and the pharmaceutical industry, a need for large-scale production of flagellin is anticipated. Traditionally, flagellin has been purified on the laboratory scale by differential ultracentrifugation after chemical or mechanical shearing of bacteria to release the flagella from the resuspended bacterial pellets (Ibrahim et al. 1985; Kelly-Wintenberg et al. 1990; Brett et al. 1994; Montie and Stover 1983). In these protocols, a key step in maximising the yield is the prevention of 
flagellar shearing during bacterial culture and the generation of the bacterial pellet. These steps were traditionally achieved by growing bacteria with shaking at low speed or in static cultures. In the present work, we describe a method suitable for scaling up flagellin production to several hundred litres using high-yield aerated and agitated bacterial culture conditions and producing high yields of purified flagellin per litre of culture.

\section{Materials and methods}

Strains and reagents

The bacterial strain employed was Salmonella enterica serovar typhimurium SIN22 (Sierro et al. 2001). Chemicals were from Sigma (St. Louis, USA), unless otherwise stated.

Culture conditions and purification protocol

Five millilitres of TSB medium (Merck Cat. No. 1.05459, Germany) was inoculated and grown for $6 \mathrm{~h}$. One millilitre of this culture was diluted in $200 \mathrm{ml}$ of fresh medium and grown overnight. This culture was used to inoculate 101 of TSB medium under the following conditions: (1) static culture for $72 \mathrm{~h}$ at $37^{\circ} \mathrm{C}$ (2) aerated in a glass laboratory bioreactor for $3 \mathrm{~h}$ at $37^{\circ} \mathrm{C}$ with an air flow rate of $10 \mathrm{l} / \mathrm{min}$ without agitation or (3) aerated and agitated at $420 \mathrm{rev} / \mathrm{min}$ in a bioreactor for $3 \mathrm{~h}$ at $37^{\circ} \mathrm{C}$ with an air flow rate of $10 \mathrm{l} /$ $\min$.

To evaluate the convenience of combining cross-flow filtration (CFF) with downstream processing, a static culture was concentrated using cross-flow filtration to obtain 11 of bacterial culture (Hollow Fibre Cartridge with $2,800 \mathrm{~cm}^{2}$ of surface area and $0.1 \mu \mathrm{m}$ pore diametre, Model\# CFP-1-E-6A, GE Healthcare, Germany and a peristaltic pump, Model 620S, Watson-Marlow, Brazil). The concentrated bacterial suspension was separated into three fractions that were processed differently to release their flagella. One fraction of $200 \mathrm{ml}$ was sheared using vortex agitation for $2 \mathrm{~min}$ at $30 \mathrm{~Hz}\left(2 \mathrm{X}^{3}\right.$, VELP Scientifica, Italy). A second aliquot of $400 \mathrm{ml}$ was sheared using orbital shaking at $200 \mathrm{rev} / \mathrm{min}$ for $5 \mathrm{~min}$ (M-23, Vicking, Argentina) and a third $(400 \mathrm{ml})$ was ultracentrifuged directly without extra shearing to evaluate the degree of flagellar release achieved by the cross-flow filtration process. In all cases, after shearing and before ultracentrifugation, a bacterial pellet was obtained by centrifugation at $5,000 \mathrm{rev} / \mathrm{min}$ for $30 \mathrm{~min}$ (Himac CR22GII centrifuge with a R9A rotor, Hitachi Co., Japan). Next, the flagellar pellet was recovered after ultracentrifugation of the cell-free supernatant $\left(40,000 \mathrm{rev} / \mathrm{min}\right.$ for $1.5 \mathrm{~h}$ at $4^{\circ} \mathrm{C}$ in a Beckman ultracentrifuge, Fullerton, CA, USA). The flagellar pellet was resuspended in PBS and centrifuged at 5,000 rev/min for $30 \mathrm{~min}$ at $4^{\circ} \mathrm{C}$ (Centrifuge 5804, R. Eppendorf, Germany) to eliminate LPS and other bacterial debris. A clear supernatant containing flagellin was recovered for further processing and analysis. The protein contents of the different aliquots taken during the process were evaluated with the $\mathrm{BCA}^{\mathrm{TM}}$ Protein Assay Kit (Pierce, Rockford, IL, USA).

\section{SDS-PAGE and MALDI-TOF analysis}

The peptide mass fingerprint (PMF) technique was used for protein analysis. Protein purity was evaluated by SDSPAGE (Laemmli 1970). The resulting bands were recovered and rinsed three times with acetonitrile, then air dried and carbamide-methylated by reduction with $5 \mathrm{mM}$ DTT in ammonium bicarbonate and incubated for $30 \mathrm{~min}$ at 56 to $60^{\circ} \mathrm{C}$. Next, they were treated with $55 \mathrm{mM}$ iodoacetamide in $50 \mathrm{mM}$ sodium bicarbonate and digested with $5 \mu \mathrm{l}$ of $0.02 \mathrm{mg} / \mathrm{ml}$ trypsin for $30 \mathrm{~min}$ at $4^{\circ} \mathrm{C}$. This extract was mixed with $0.4 \mathrm{~g}$ acid matrix $\alpha$-cyano-4-hydroxicinnamic acid/1 of $0.5 \%$ trifluoroacetic acid in a 1:1 ratio of bi-distilled water: acetonitrile. We then placed a $0.4 \mu \mathrm{l}$ aliquot in an AnchorChip 384/600 (Bruker, Billerica, MA, USA). The samples were analysed in the Ultraflex II TOF mass spectrometer (Bruker, Billerica, MA, USA) in the positive reflectron mode, recording the intensity of peaks between 800 and 3,000 Da for analysis. To determine the sequence corresponding to the peaks, the Bio-Tools and Mascot programs were employed.

\section{LPS detoxification}

The flagellin was detoxified using polymyxin B (DetoxiGel AffinityPak ${ }^{\text {TM }}$ Pre-packed Columns 20,344, Pierce, Rockford, IL, USA). Endotoxin levels $\left(2.5 \times 10^{-3} \mathrm{UE} / \mu \mathrm{g}\right.$ FliC) were determined by the Limulus Amoebocyte Lysate (LAL) gel clot assay according to the manufacturer's instructions (Charles River Endosafe ${ }^{\circledR}$, San Diego, CA, USA). The LAL Reagent and Control Standard Endotoxin from $E$. coli strain 055:B5 were used for the Gel-Cloth Technique (Charles River Endosafe ${ }^{\circledR}$, San Diego, CA, USA).

\section{Control of biological activity}

To determine the activity of the flagellin, the in vitro and in vivo assays below were performed. A trypsin-digested flagellin preparation obtained as described was used as a control. Briefly, purified flagellin was treated for $2 \mathrm{~h}$ at $37^{\circ} \mathrm{C}$ with 5\% Trypsin-EDTA $10 \mathrm{X}$ (GIBCO, Canada), followed by a $10 \mathrm{~min}$ inactivation at $80^{\circ} \mathrm{C}$. 
Biological activity assay in vitro

The reporter cell line Caco-CCL20-luciferase was used as described (Nempont et al. 2008). Briefly, cells in 48-well plates were stimulated for $6 \mathrm{~h}$ with the indicated amounts of flagellin or flagellin treated with trypsin. Then cell lysates were prepared. The luciferase activity was measured using the Luciferase Assay System (Promega, Madison, WI, USA) and a luminometer (Labsystems, Finland), following manufactures's instructions. The fold induction of luciferase activity was calculated as the ratio of the relative luminiscence units read in the luminometer between the test condition and the unstimulated control. At least three independent wells were used for each measurement and average results were used to calculate the ratio, as previously described (Nempont et al. 2008).

\section{Pyrogenic tests on rabbits}

Pyrogen testing was performed and evaluated according to USP $31 / \mathrm{NF} 26<151>$. Three rabbits per group were injected with $100 \mu \mathrm{g} / \mathrm{kg}$ of body weight of FliC or detoxified FliC, and any temperature increase was evaluated.

\section{Induction of the innate response in vivo}

Assays were performed as described previously (Rumbo et al. 2004; Nempont et al. 2008). Briefly, Balb/c mice were stimulated with either $10 \mu \mathrm{g}$ flagellin in $300 \mu$ pyrogen-free PBS via intraperitoneal (IP) injection or $1 \mu \mathrm{g}$ flagellin in $50 \mu$ pyrogen-free PBS by the intranasal (IN) route under slight ether anaesthesia. After $2 \mathrm{~h}$, the animals were euthanized by cervical disruption, and a portion of the small intestine was taken from mice stimulated IP and the left lung was taken from mice stimulated IN. The organs were processed for total RNA extraction using the illustra RNA spin mini kit (GE Healthcare, Germany) followed by Dnase I treatment according to the manufacturer's instructions. Reverse transcription used $1 \mu \mathrm{g}$ of RNA and the M-MLV reverse transcriptase (Invitrogen, Carlsbad, CA, USA). The cDNA was PCR-amplified using the following primers: CCL20 fwd: 5'TTTTGGGATGGAATTGGACAC3' ${ }^{\prime}$ and rev: $5^{\prime}$ TGCAGGTGAAGCCTTCAACC $3^{\prime}$ and $2 \mathrm{X} \mathrm{iQ}^{\mathrm{TM}}$ SYBR Green Supermix (Bio-Rad, Hercules, CA, USA). Denaturation was $5 \mathrm{~min}$ at $95^{\circ} \mathrm{C}$ followed by 40 cycles of amplification at $95^{\circ} \mathrm{C}$ for $30 \mathrm{~s}$ and $60^{\circ} \mathrm{C}$ for $30 \mathrm{~s}$ using the iCycler (BioRad, USA). Fold induction was calculated using the $\Delta \Delta \mathrm{Ct}$ method as described (Anderle et al. 2005).

\section{Statistical analysis}

Differences among means were assessed by two tailed Student's $t$ tests with significance accepted at the $P<0.05$ level.

\section{Results}

The use of CFF allows the recovery of flagellin from concentrated culture supernatant.

To manage a high volume of bacterial culture, we tested the use of CFF as the first step in concentrating bacterial cultures prior to separation of the pellet by centrifugation. We produced 101 of Salmonella culture in static batch bottles to minimize the shearing of flagella from bacteria, as is usually recommended in conventional protocols (Ibrahim et al. 1985; Kelly-Wintenberg et al. 1990; Brett et al. 1994; Montie and Stover 1983).

To evaluate the convenience of combining CFF with different shearing protocols in the downstream processing of bacterial cultures, several alternatives were tested (Fig. 1a). In all cases, upon ultracentrifugation, a single band identified as flagellin by MALDI-TOF analysis was recovered (Fig. 1b). We were able to directly recover flagellin upon ultracentrifugation of the CFF concentrate without any extra shearing step (condition 2 in Fig. 1a scheme). According to the different yields obtained (Fig. 1d), the CFF produced extensive flagellar shearing because the recovery of flagellin in the CFF without a shearing step was about $90 \%$ of that obtained with a similar protocol but including an extra mechanical shearing step. In similar experiments, after static Salmonella growth and mechanical shearing without a CFF step, following the traditional lab-scale protocols on the same bulk culture, the protein yield was approximately half of that obtained in this experiment (data not shown). This indicates that the CFF step possibly improves the yield by producing extensive flagellar shearing. In all cases, the flagellin obtained showed similar capacities to induce the activation of the reporter cell line Caco-CCL20-luciferase (Fig. 1c). These results also indicate that, after CFF, most of the flagellin has been released from the bacteria but is retained in the concentrated bacterial supernatant. Coincidently with this result, no Caco-CCL20-luciferase stimulating activity was detected in the flow-through obtained after the CFF step (data not shown), indicating that this process retains all of the bioactive flagellin in the concentrate.

The optimized protocol allows the use of non-restricted bacterial culture conditions.

Because CFF is able to concentrate bacterial cultures and retain the flagellin released from the bacterial surface, we tested different bacterial growing conditions in aerated, agitated fermentators that allow a high yield of bacterial growth despite causing flagellar shearing (Table 1). After bacterial growth, the cultures were processed directly by $\mathrm{CFF}$ and ultracentrifugation (similar to condition 2 in Fig. 1a scheme). The results indicate that the culture conditions that result in maximal bacterial growth are also the ones that produce the best yield (in flagellin/l of culture) with the selected protocol. It is remarkable that the combination of high aeration and agitation conditions with CFF increases the 
A

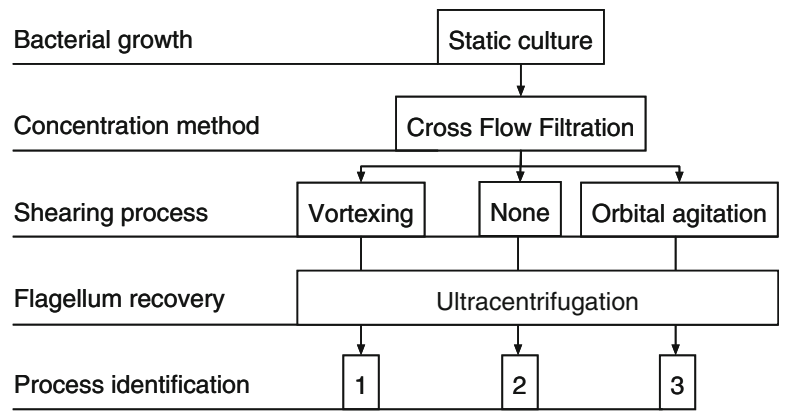

B

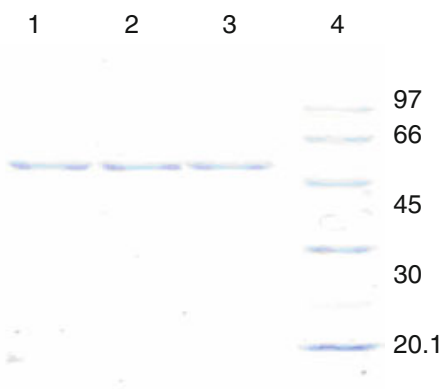

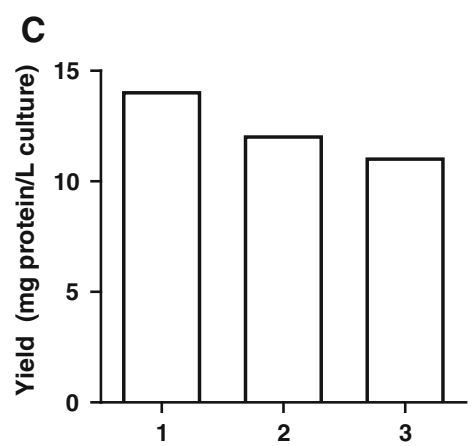

Fig. 1 a A comparison of the schemes of different protocols. The same bulk culture was processed in parallel, according to the scheme, resulting in three different procedures. The main differences among them are related to the shearing process. In process no. 1, shearing was performed on a concentrated bacterial culture by vortexing; in process no. 2, no shearing operation was performed; and in process no. 3, shearing was performed by $5 \mathrm{~min}$ of orbital agitation at $200 \mathrm{rpm}$. b SDS-PAGE of the proteins obtained from the three different procedures. The bands were recovered and analysed by MALDI-TOF. Lanes 1 to 3 are flagellins. Lane number is coincident with the process number indicated in (a). Lane 4 is a molecular

Table 1 Yields of different bacterial growing conditions

\begin{tabular}{llll}
\hline Culture type & $\begin{array}{l}\text { Culture } \\
\text { conditions }\end{array}$ & Final DO & $\begin{array}{l}\text { Protein } \\
\text { yield }\end{array}$ \\
\hline Static culture & $72 \mathrm{~h} 37^{\circ} \mathrm{C} 35-1$ & 1.27 & $12 \pm 0.5$ \\
$\begin{array}{l}\text { Aerated/agitated } \\
\text { culture }\end{array}$ & $\begin{array}{l}2 \mathrm{~h} 45^{\prime} 37^{\circ} \mathrm{C} 10-1 \\
10-1 / \mathrm{min}\end{array}$ & 2.8 & $29.9 \pm 0.8 \mathrm{mg} / \mathrm{l}$ \\
& $420 \mathrm{rev} / \mathrm{min}$ & & \\
Aerated/culture & $3 \mathrm{~h} 37^{\circ} \mathrm{C} 10-1$ & 2.5 & $20.0 \pm 0.9 \mathrm{mg} / 1$ \\
& $10-1 / \mathrm{min} 2.5$ & &
\end{tabular}

The highest yield in bacteria match the condition that also produce the best yield in flagellin

yield of flagellin/l of bacterial culture at least twofold compared to the yield obtained with the static cultures used in our previous experiment. Furthermore, the yield of flagellin from this optimized protocol is fourfold higher than the yield obtained with the same bacterial strain in preliminary experiments following the usual lab-scale protocol (not shown).

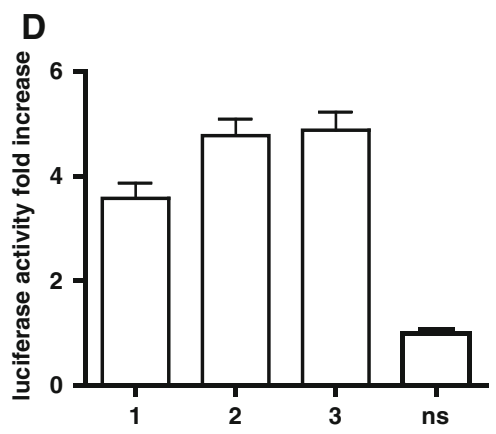

weight marker. Mass in $\mathrm{kDa}$ is indicated in the figure. $\mathbf{c}$ The yields of different processes. Process number is according to (a). Flagellin recovery from cross-flow filtration without a shearing step was $90 \%$ of that obtained when an extra mechanical shearing step was included. d The biological activity assay on the reporter cell line Caco-CCL20-luciferase. Cells in 48-well plates were stimulated with $1 \mu \mathrm{g} / \mathrm{ml}$ of flagellins obtained from processes 1,2 or 3 as described in (a). The relative luciferase activity is shown as the mean $\pm \mathrm{SD}$ of triplicate analysis. Results are representative from three different experiments. The non-stimulated condition (ns) was assigned a relative value of 1

The flagellin obtained by the large-scale flagellin production protocol has comparable bioactivity to that obtained by the laboratory-scale protocol.

To check that the biological properties of the flagellin produced by the lab-scale methodology and the improved, scaled-up protocol are equivalent, a fraction of each lot was detoxified using polymyxin columns. The elimination of endotoxin activity was evidenced by the results of the pyrogenicity test and the bioactivity of crude and trypsintreated fractions (Fig. 2).

One of the key features of flagellin is its capacity to trigger innate immune response (Rumbo et al. 2006). Consequently, to evaluate the activity of the flagellins produced either at laboratory scale or using our scalable protocol, we used either systemic or mucosal delivery of flagellin and determined the trasncriptional response at different locations, following protocols usually employed in our laboratory. The bioactive properties of the flagellin produced by the laboratory-scale and scaled-up protocols were comparable because both preparations were able to 

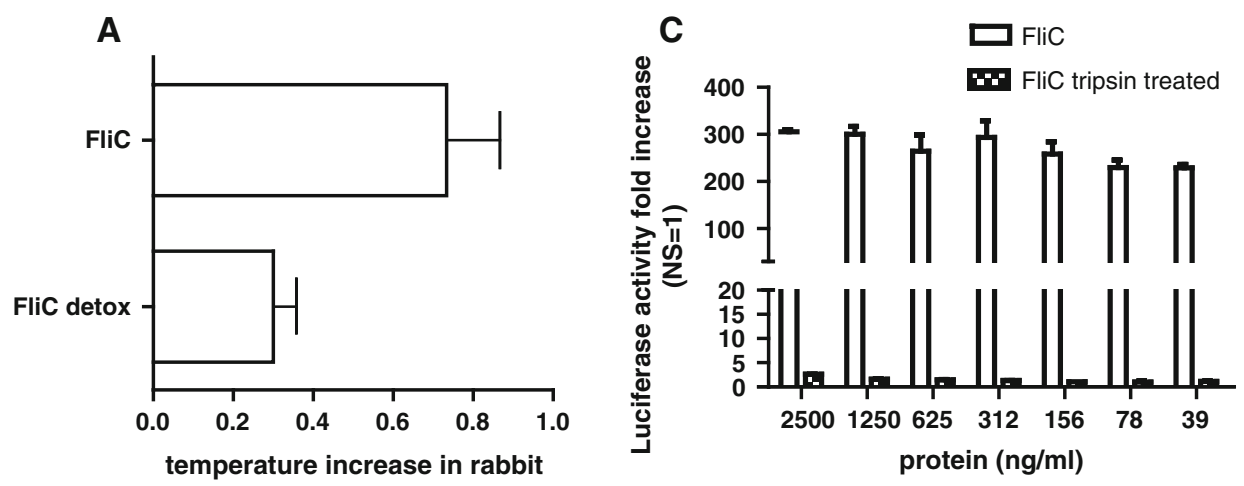

B Limulus Amebocyte Lysate
\begin{tabular}{|l|l|}
\hline Sample & Result \\
\hline FliC ccf & positive \\
\hline FliC ccf detox & negative \\
\hline
\end{tabular}

Fig. 2 In vitro and in vivo assays to test the efficiency of the LPS depletion process. a The pyrogen test USP 27/NF $22<151>$ performed in rabbits. Three animals per group were treated with $100 \mu \mathrm{g} / \mathrm{kg}$ of crude or detoxified FliC, and changes in temperature were noted after 1,2 and $3 \mathrm{~h}$. The maximum increase in temperature as a result of the test is noted for each animal. Samples that induced a rise in temperature higher that $0.6^{\circ} \mathrm{C}$ were considered pyrogenic. b The Limulus test (gel clot method) USP 27/NF $22<85>$

induce a CCL20 response in the liver (not shown) and intestine when delivered by the IP route (Fig. 3). The preparations were also able to induce CCL20 expression in the lung when delivered by the IN route (Fig. 3).

\section{Discussion}

The recovery of flagella from culture supernatants by ultracentrifugation is a simple way of producing purified flagellin and has been used for decades (Ibrahim et al. 1985; Kelly-Wintenberg et al. 1990; Brett et al. 1994; Montie and Stover 1983). However, it has two main drawbacks for the processing of large amounts of bacterial culture. There is the need to keep the intact flagellum adhered to the bacteria until the shearing step imposes restrictions on the culture conditions, preventing the production of high yields of bacteria by using highly aerated and agitated conditions. The use of this protocol for the large-scale production of flagellin imposes a need for continuous-flow centrifuges for the concentration of bacterial culture to achieve volumes compatible with the flagellar-shearing step and the downstream ultracentrifugation step. To scale up flagellin production and avoid these drawbacks, we have developed a protocol using CFF as a downstream step after bacterial growth. This protocol avoids the problems mentioned above, because the CFF t performed on crude flagellin obtained by procedure 2 and on the same preparation detoxified using polymyxin columns. c The luciferase activity assay stimulated by FliC and trypsin-treated FliC. The cell reporter line Caco-CCL20-luciferase was stimulated for $6 \mathrm{~h}$ and luciferase activity was measured in a luminometer. The relative luciferase activity is shown as the mean $\pm \mathrm{SD}$ of triplicate analysis. Results are representative from three different experiments. The nonstimulated condition (ns) was assigned a relative value of 1

allows concentration of the bacterial culture in the same step and induces shearing that is at least as effective as the mechanical shearing usually used in low-scale protocols. This allows the elimination of any further shearing step and permits a straightforward processing of the culture to obtain the bacterial pellet and separate the flagella from the supernatant by ultracentrifugation. This results in a simple protocol with the advantage that the cross-flow filtration step is flexible and easy to adapt to different industrial settings, because it does not necessitate any expensive equipment and allows the combination of steps dealing with different volumes. Although in our experiments this step allowed us to concentrate bacterial cultures around tenfold, it may be possible to enhance this concentration procedure several times more. Furthermore, this procedure has, in principle, no limitations on the volume of batch culture to be used, being highly flexible in combining steps with a high volume of bacterial culture with low-volume downstream steps (Saxena et al. 2009). There are several CFF cartridges with different sizes, allowing the adoption of the procedure for a variety of purposes, from small-scale laboratory research to industrial applications.

Beyond these operational advantages, one major improvement inherent in our protocol is that the CFF step eliminates the restriction of growing the bacteria in lowshearing conditions to avoid flagella lost in the culture supernatant. Although the pore size of the CFF device used 

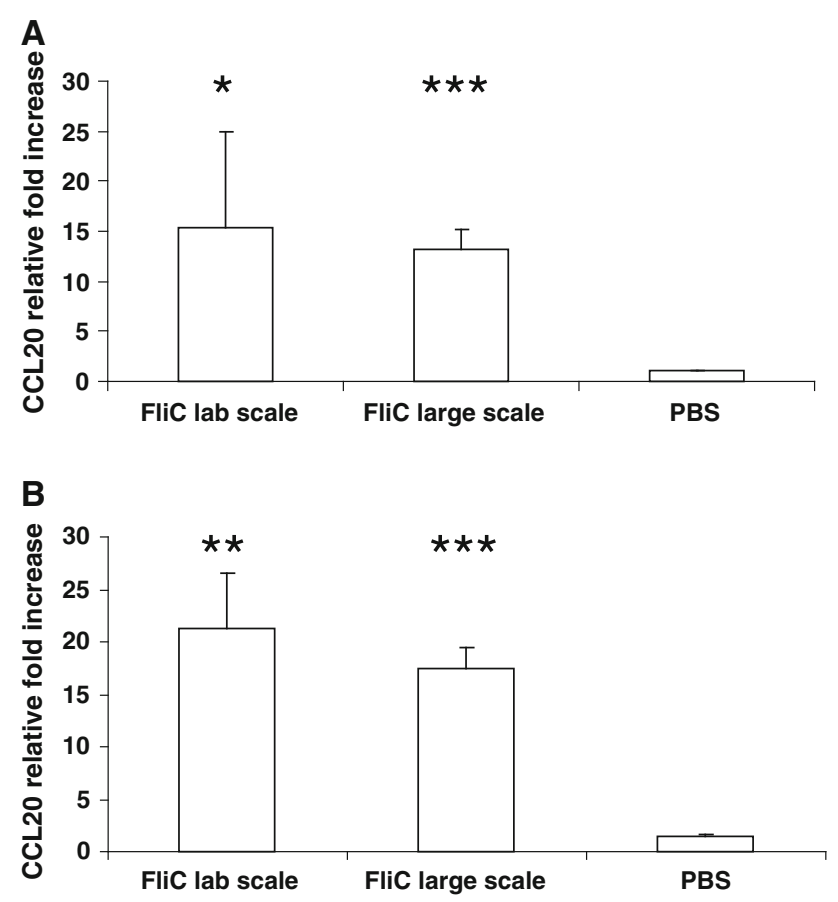

Fig. 3 The flagellin obtained by the large-scale production protocol has comparable bioactivity to that obtained by the laboratory-scale protocol. a Stimulation of CCL20 expression in murine intestine. The mice were stimulated intraperitoneally with $10 \mu \mathrm{g}$ of detoxified flagellin in pyrogen-free PBS, and $2 \mathrm{~h}$ later the relative expression of CCL20 was evaluated by qPCR $(n=3)$. The results are representative of 3 independent experiments. b The stimulation of CCL20 expression in murine lung. Mice were stimulated with $1 \mu \mathrm{g}$ of detoxified flagellin in PBS by the intranasal route. The left lung was removed and relative CCL20 expression levels were analysed by qPCR $(n=3)$. The results are representative of 2 independent experiments. Statistical differences with the PBS control are indicated. $* P \leq 0.05, * * P \leq 0.01, * * * P \leq 0.001$. No statistical differences were found between the different flagellin stimulation assayed

is not small enough to retain free flagellin, the highly polymeric structure of the flagella imposes steric constraints that result in the complete retention of the flagellin in the CFF concentrate, as has been demonstrated using a highly sensitive bioassay to detect flagellin-dependent TLR5 stimulation (Nempont et al. 2008). This finding enabled us to select culture conditions that allow a much higher yield in bacterial growth regardless of the eventual shearing of the flagella during the culture. This allowed us to increase around four-fold the yield of flagellin production/l of bacterial culture, as compared to traditional protocols using the same strain. For the present study we have used Salmonella enterica serovar typhimurium SIN22, that was employed in several studies as source of flagellin using as control an isogenic strain carrying a deletion on the flagellin gene (Sierro et al. 2001). Although not extensively studied, we have applied the proposed protocol to other Salmonella strains, recovering flagellin with comparable yield and purity. In principle, the developed procedure could be applied to different bacterial strains and would allow to obtained improved yield with a process that is simpler than the traditional procedure. Furthermore, different growing conditions and culture medium supplements could be tested to increase the biomass yield and improve the flagellin yield. Among the conditions tested, we have observed that growing at highly aerated and agitated conditions results in a yield (Table 1) that is higher than in other conditions, even when it is normalized by DO. This implies that the highly aerated and agitated conditions allowed a better yield of bacteria/L of culture but also increased the amount of flagellin produced per bacterium, possibly due to enhanced flagellin synthesis induced by flagella lost due to agitation during growth in high-shearing conditions. This effect can be exploited by the use of the CFF protocol described here and could be enhanced by even more vigorous culture conditions.

In our procedure, endotoxin removal was performed using prepacked polymyxin B columns. Depending on the scale of production, batch extractions may be possible. This process was efficiently performed, according to the in vivo and in vitro tests for endotoxin activity (Fig. 2). Although SDS-PAGE analysis and MS fingerprinting were indicative of a high purity of the flagellin obtained, we could not exclude the presence of minor contaminants. The proposed protocol can be combined with different downstream purification steps to assure the complete elimination of potential contaminants, depending on the end use of the flagellin. Several bioassays indicated that the properties of the flagellin obtained by these different methods are equivalent. Overall, these results indicate that the protocol described in this work can be envisaged as an efficient means of massive production of flagellin for several biotechnological uses, such as adjuvants for immunization protocols (Mizel and Bates 2010) or radiotherapies (Jones et al. 2011), boosters of granulopoiesis (Burdelya et al. 2008) or vaccine antigens (McNeilly et al. 2008).

In conclusion, we have developed a simple, high-yield protocol to scale up flagellin purification procedures that can be adopted in industrial settings without the necessity of sophisticated or expensive equipment.

Acknowledgments This work was supported by grant INCO FP6CT 032296 to National University of La Plata and BIOL SAIC to MR and JCL. YH is a fellow from Consejo Nacional de Investigaciones Científicas y Técnicas (CONICET). AE is a fellow from ANPCYT. MR is a member of the Scientific Carreer of CONICET.

Conflict of interest The authors declare that they have no conflict of interest. 


\section{References}

Anderle P, Rumbo M, Sierro F, Mansourian R, Michetti P, Roberts MA, Kraehenbuhl JP (2005) Novel markers of the human follicle-associated epithelium identified by genomic profiling and microdissection. Gastroenterology 129(1):321-327

Brett PJ, Mah DC, Woods DE (1994) Isolation and characterization of Pseudomonas pseudomallei flagellin proteins. Infect Immun 62(5):1914-1919

Burdelya LG, Krivokrysenko VI, Tallant TC, Strom E, Gleiberman AS, Gupta D, Kurnasov OV, Fort FL, Osterman AL, Didonato JA, Feinstein E, Gudkov AV (2008) An agonist of toll-like receptor 5 has radioprotective activity in mouse and primate models. Science 320(5873):226-230

Honko AN, Sriranganathan N, Lees CJ, Mizel SB (2006) Flagellin is an effective adjuvant for immunization against lethal respiratory challenge with yersinia pestis. Infect Immun 74(2):1113-1120

Ibrahim GF, Fleet GH, Lyons MJ, Walker RA (1985) Method for the isolation of highly purified salmonella flagellins. J Clin Microbiol 22(6): 1040-1044

Jones RM, Sloane VM, Wu H, Luo L, Kumar A, Kumar MV, Gewirtz AT, Neish AS (2011) Flagellin administration protects gut mucosal tissue from irradiation-induced apoptosis via MKP-7 activity (in press)

Kelly-Wintenberg K, Anderson T, Montie TC (1990) Phosphorylated tyrosine in the flagellum filament protein of Pseudomonas aeruginosa. J Bacteriol 172(9):5135-5139

Laemmli UK (1970) Cleavage of structural proteins during the assembly of the head of bacteriophage t4. Nature 227:680-685

Lane MC, Alteri CJ, Smith SN, Mobley HL (2007) Expression of flagella is coincident with uropathogenic escherichia coli ascension to the upper urinary tract. Proc Natl Acad Sci USA 104(42): 16669-16674. Epub 12007 Oct 16669

Lee SE, Kim SY, Jeong BC, Kim YR, Bae SJ, Ahn OS, Lee JJ, Song HC, Kim JM, Choy HE, Chung SS, Kweon MN, Rhee JH (2006) A bacterial flagellin, vibrio vulnificus flab, has a strong mucosal adjuvant activity to induce protective immunity. Infect Immun 74(1):694-702

Lightfield KL, Persson J, Brubaker SW, Witte CE, von Moltke J, Dunipace EA, Henry T, Sun YH, Cado D, Dietrich WF, Monack DM, Tsolis RM, Vance RE (2008) Critical function for naip5 in inflammasome activation by a conserved carboxy-terminal domain of flagellin. Nat Immunol 9(10): 1171-1178. Epub 2008 Aug 1124

Mahajan A, Currie CG, Mackie S, Tree J, McAteer S, McKendrick I, McNeilly TN, Roe A, La Ragione RM, Woodward MJ, Gally
DL, Smith DG (2009) An investigation of the expression and adhesin function of h7 flagella in the interaction of Escherichia coli O157: H7 with bovine intestinal epithelium. Cell Microbiol 11(1):121-137

McNeilly TN, Naylor SW, Mahajan A, Mitchell MC, McAteer S, Deane D, Smith DG, Low JC, Gally DL, Huntley JF (2008) Escherichia coli O157:H7 colonization in cattle following systemic and mucosal immunization with purified h7 flagellin. Infect Immun 76(6):2594-2602

Miao EA, Andersen-Nissen E, Warren SE, Aderem A (2007) Tlr5 and ipaf: dual sensors of bacterial flagellin in the innate immune system. Semin Immunopathol 29(3):275-288

Mizel SB, Bates JT (2010) Flagellin as an adjuvant: cellular mechanisms and potential. J Immunol 185(10):5677-5682

Montie TC, Stover GB (1983) Isolation and characterization of flagellar preparations from Pseudomonas species. J Clin Microbiol 18(3):452-456

Nempont C, Cayet D, Rumbo M, Bompard C, Villeret V, Sirard JC (2008) Deletion of flagellin's hypervariable region abrogates antibody-mediated neutralization and systemic activation of tlr5dependent immunity. J Immunol 181(3):2036-2043

Rumbo M, Sierro F, Debard N, Kraehenbuhl JP, Finke D (2004) Lymphotoxin beta receptor signaling induces the chemokine ccl20 in intestinal epithelium. Gastroenterology 127(1):213-223

Rumbo M, Nempont C, Kraehenbuhl JP, Sirard JC (2006) Mucosal interplay among commensal and pathogenic bacteria: lessons from flagellin and toll-like receptor 5. FEBS Lett 580(12): 2976-2984. Epub 2006 Apr 2921

Saxena A, Tripathi BP, Kumar M, Shahi VK (2009) Membrane-based techniques for the separation and purification of proteins: an overview. Adv Colloid Interface Sci 145(1-2):1-22

Sierro F, Dubois B, Coste A, Kaiserlian D, Kraehenbuhl JP, Sirard JC (2001) Flagellin stimulation of intestinal epithelial cells triggers ccl20-mediated migration of dendritic cells. Proc Natl Acad Sci USA 98(24):13722-13727

Skountzou I, Martin MD, Wang B, Ye L, Koutsonanos D, Weldon W, Jacob J, Compans RW (2010) Salmonella flagellins are potent adjuvants for intranasally administered whole inactivated influenza vaccine. Vaccine 28(24):4103-4112

Strindelius L, Filler M, Sjoholm I (2004) Mucosal immunization with purified flagellin from salmonella induces systemic and mucosal immune responses in $\mathrm{c} 3 \mathrm{~h} / \mathrm{hej}$ mice. Vaccine 22(27-28): $3797-3808$ 